\title{
Tuning Spatial Profiles of Selection Pressure to Modulate the Evolution of Resistance
}

\author{
Maxwell G. De Jong ${ }^{1}$ and Kevin B. Wood ${ }^{1,2}$ \\ ${ }^{1}$ Department of Physics, University of Michigan, Ann Arbor, Michigan 48109, USA \\ ${ }^{2}$ Department of Biophysics, University of Michigan, Ann Arbor, Michigan 48109, USA
}

\begin{abstract}
Spatial heterogeneity plays an important role in the evolution of drug resistance. While recent studies have indicated that spatial gradients of selection pressure can accelerate resistance evolution, much less is known about evolution in more complex spatial profiles. Here we use a stochastic toy model of drug resistance to investigate how different spatial profiles of selection pressure impact the time to fixation of a resistant allele. Using mean first passage time calculations, we show that spatial heterogeneity accelerates resistance evolution when the rate of spatial migration is sufficiently large relative to mutation but slows fixation for small migration rates. Interestingly, there exists an intermediate regime - characterized by comparable rates of migration and mutation - in which the rate of fixation can be either accelerated or decelerated depending on the spatial profile, even when spatially averaged selection pressure remains constant. Finally, we demonstrate that optimal tuning of the spatial profile can dramatically slow the spread and fixation of resistant subpopulations, even in the absence of a fitness cost for resistance. Our results may lay the groundwork for optimized, spatially-resolved drug dosing strategies for mitigating the effects of drug resistance.
\end{abstract}

Drug resistance is a rapidly growing public health threat and a central impediment to the treatment of cancer, viruses, and microbial infections [1 $[4]$. The battle against resistance has been largely fought at the molecular level, leading to an increasingly mature understanding of its underlying biochemical and genetic roots. At the same time, evolutionary biologists have long recognized resistance as a fundamentally stochastic process governed by the complex interplay between microbial ecology and evolutionary selection. The last decade, in particular, has seen a significant surge in efforts to develop and understand evolution-based treatment strategies to forestall resistance $[5-16]$. While the vast majority of this work focuses on spatially homogeneous environments, a number of recent studies, both theoretical and experimental, have demonstrated that spatial heterogeneity in drug concentration can dramatically alter the evolutionary dynamics leading to resistance [16 24]. On a practical level, the picture that emerges is somewhat bleak, as resistance evolution is dramatically accelerated in the presence of spatial gradients in drug concentration 18 20, 22 24] or heterogeneous drug penetration [17, 21]. Interestingly, however, recent work shows that this acceleration can be tempered or even reversed when the mutational pathway (i.e. the genotypic fitness landscape) leading to resistance contains fitness valleys [18], which are known to inhibit evolution 25 28]. Unfortunately, because the fitness landscape is a genetic property of the cells themselves, the potential for accelerated evolution appears to be "built in", making it difficult to combat in a treatment setting. However, these results raise the question of whether non-monotonic profiles of tunable properties of the system - for example, the spatial selection pressuremight also have the potential to slow evolution, even when the mutational pathway lacks the requisite fitness valleys.

Evolution in natural or clinical settings takes place in heterogeneous environments characterized by spatial fluctuations in multiple factors, including drug concentrations, nutrients, temperature, and $\mathrm{pH}$, all of which potentially affect cellular growth. Understanding evolution and ecology in such spatially extended systems is a challenging and long-studied problem 29 33]. Recent studies have demonstrated rich dynamics when inter-cellular interactions are defined on heterogeneous complex networks 34 36], where spatial structure can (for example) promote invasive strategies in tumor models [35] or modulate fixation times on random landscapes [34]. Remarkably, in the weak selection limit, evolutionary dynamics can be solved for any population structure 36], providing extensive insight into game-theoretic outcomes on complex networks. In addition, theoretical tools from statistical physics have proven useful for understanding spatiotemporal dynamics in spatially structured populations in a wide range of contexts, including biologicallyinspired Monte Carlo models [18], toy models of sourcesink dynamics [19], stepping-stone models of spatial pattern formation [37], models of dispersion [38-42], and Moran meta-population models [43 45]. In a similar spirit, here we use stochastic models of evolution along with theoretical tools from statistical physics to investigate the effects of spatially heterogeneous fitness pressures on the evolution of resistance. In contrast to previous models defined on heterogeneous networks at the single-cell level, here we consider meta-populations connected via simple topologies and investigate the effects of spatial structure imposed by arbitrary distributions of selection pressure. While several elegant approaches exist for studying these models in particular limits (e.g. with a center manifold reduction) [43 45], here we instead use a classical mean first passage time approach based on adjoint equations to reduce the calculation of mean fixation times to a simple collection of linear equations that can be easily solved for arbitrary spatial distri- 
butions of selection pressures. This method also allows us to find the fixation times from arbitrary initial states, which are often difficult to compute using other methods. Using this approach, we show that resistance evolution can be either accelerated or decelerated by spatial heterogeneities in selection pressure, even when the spatially averaged selection pressure remains constant. In addition, we demonstrate that tuning the spatial distribution of selection pressure can dramatically slow fixation when the subpopulations of resistant mutants are not uniformly distributed in space.

To investigate resistance evolution on a spatially heterogeneous landscape, we consider a stochastic Moranlike model [46] of a finite population $(N)$ consisting of $\left(N-n^{*}\right)$ wild-type cells with fitness $r_{0} \leq 1$ and $n^{*}$ drugresistant mutants with fitness $r^{*}$, which we set to unity without loss of generality. Note that this model does not include a fitness cost to resistance (i.e. $r^{*} \geq r_{0}$ for all conditions). At each time step, cells are randomly selected for birth and death, with cells of higher fitness (in this case, resistant cells) chosen preferentially for division (see SI for full model with transition rates). Wildtype cells can mutate to become drug resistant at rate $\mu$; we neglect reverse transitions to the drug-sensitive state. To incorporate spatial heterogeneity, we consider a simple spatially extended system with $M$ distinct microhabitats, each containing $N$ cells; cells are allowed to migrate at rate $\beta$ between connected microhabitats (Fig. 1a). At each time $t$, the state of the system is characterized by the vector $n^{*}\left(x_{i}\right)$ whose components correspond to the number of mutants in each discrete microhabitat $x_{i}=0,1, \ldots, M-1$. The system evolves according to a continuous time master equation

$$
\frac{d P_{m}}{d t}=\sum_{m^{\prime}} \Omega_{m m^{\prime}} P_{m^{\prime}}
$$

where $m$ and $m^{\prime}$ denote different states of the system and $\Omega$ is a $N^{M} \times N^{M}$ matrix whose entries depend on the wild-type fitness value $r_{0}\left(x_{i}\right)$ at each spatial location $x_{i}$ (see SI). For tractability, we restrict our analysis to $M=3$, which is the simplest model that allows for potentially non-monotonic fitness landscapes, such as fitness peaks and fitness valleys. In what follows, we refer to the vector $s\left(x_{i}\right) \equiv 1-r_{0}\left(x_{i}\right)$ as the spatial profile of selection pressure, as it measures the difference in fitness between resistant and wild-type cells in each microhabitat $\left(x_{i}\right)$. Intuitively, large values of $s\left(x_{i}\right)$ correspond to regions where the resistant mutant has a significant evolutionary advantage over the wild-type cells (e.g. regions of high drug concentration).

While Equation 1 is difficult to solve explicitly, it is straightforward to calculate quantities that describe the evolution of resistance in various spatial profiles. The model consists of a single absorbing state - the fully resistant state $\left(n^{*}\left(x_{i}\right)=N\right.$ for all $\left.x_{i}\right)$-and the system will asymptotically approach this state. To characterize the speed of fixation in the presence of different spatial profiles $s\left(x_{i}\right)$, we calculate the mean first passage times (MFTPs) between states, which obey [47, 48]

$$
-1=\sum_{m^{\prime} \neq m_{f}} T\left(m_{f} \mid m^{\prime}\right) \Omega_{m^{\prime}, m_{i}}
$$

where $T\left(m_{f} \mid m_{i}\right)$ is the mean time required for a system initially in state $m_{i}$ to first reach state $m_{f}$. We take $m_{f}$ to be the fully resistant state and solve the coupled set of linear equations for $\tau_{f}^{j} \equiv T\left(m_{f} \mid j\right)$, where $j$ is an index that runs over all initial states. In particular, when $j$ is the fully wild-type population $\left(n^{*}\left(x_{i}\right)=0\right.$ for all $\left.x_{i}\right)$, we refer to the MFPT as the mean fixation time $\tau_{f}$.

In the case of a single microhabitat, the mean fixation time $\tau_{f}$ increases as selection pressure decreases (see SI). In the spatially extended case, $\tau_{f}$ would also be expected to increase when the selection pressure is globally decreased, though it should also depend on the spatial structure of the specific selection profile $s\left(x_{i}\right)$. To investigate the impact of spatial structure alone, we compared $\tau_{f}$ across different selection profiles $s\left(x_{i}\right)$, all of which were characterized by the same spatially averaged selection pressure, $\langle s\rangle=\sum_{i} s\left(x_{i}\right) / M$. For simplicity, we begin with a symmetric profile characterized by a background selection pressure $s_{0}$ in the edge habitats and a relative peak of height $\delta s$ in the center habitat (Fig. 1a). This toy landscape has an average selection pressure of $\langle s\rangle=s_{0}+\delta s / M$, and the parameters $s_{0}$ and $\delta s$ are constrained by the fact that $0 \leq s\left(x_{i}\right) \leq 1$ at all spatial locations. We vary $\delta s$ systematically to explore different selection profiles, which can include a single selection pressure valley $(\delta s<0)$, a homogeneous landscape $(\delta s=0)$, or a single selection pressure peak $(\delta s>0)$.

Interestingly, we find that modulating heterogeneity $(\delta s)$ can increase or decrease $\tau_{f}$ for certain choices of migration and mutation rates, even when $\langle s\rangle$ is held constant (Fig. 1b). More generally, we find that the $\beta-\mu$ plane can be divided into three non-overlapping regions where the homogeneous landscape 1) leads to the smallest value of $\tau_{f}, 2$ ) leads to the largest value of $\tau_{f}$, or 3 ) does not correspond to an extremum $\tau_{f}$ (Fig. 2a-b). In the latter region, heterogeneity often modulates the fixation time by only a few percent, but we do find larger effects in the high and low migration limits (i.e. on the edges) of the intermediate regime (Fig. S1). In addition, as we increase $\beta$ for a fixed value of $\mu, \tau_{f}$ smoothly transitions from being minimized at $\delta s=0$ to being maximized near $\delta s=0$ (Fig. S1). We find empirically that the fixation time can be dominated by $\tau_{1}$, the time required to achieve a small population of mutants (Fig. 2C, rightmost panel) or $\tau_{2}$, the time required for this small population to achieve fixation (Fig. 20. leftmost panel). However, in many cases - particularly those close to the intermediate region where fixation can be accelerated or 
(a)

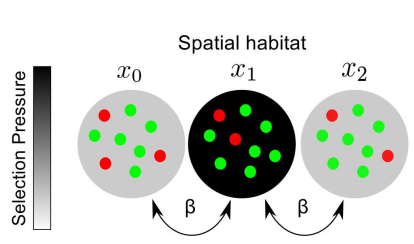

(b)

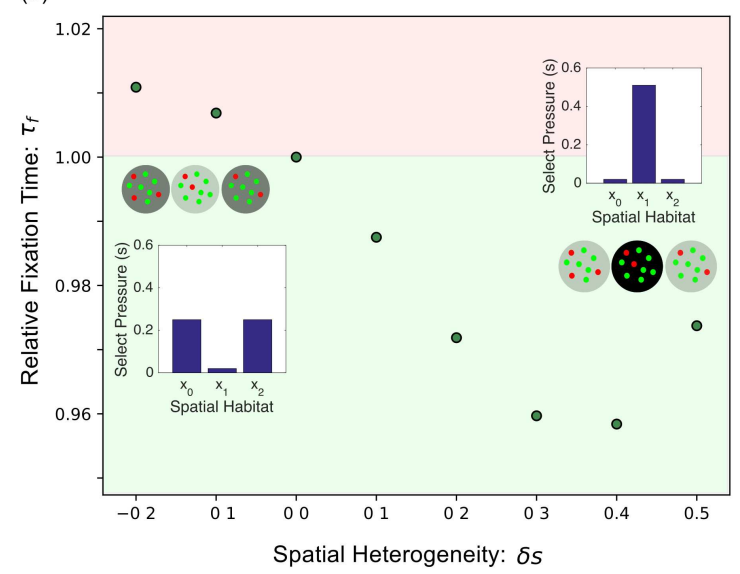

FIG. 1. (a) Stochastic model for emergence and spread of resistant cells (red) in a spatially extended population of sensitive cells (green). Each spatial habitat $\left(x_{i}\right)$ contains $N$ total cells. Cells migrate at a rate $\beta$ between neighboring habitats, and sensitive cells mutate at a rate $\mu$ to resistant cells. The spatial distribution of selection pressure is characterized by a background value $\left(s_{0}\right)$ and a peak height $(\delta s)$. (b) Example plot of the mean fixation time for different landscapes with $\mu=5 \times 10^{-3}, \beta=0.08, N=25$, and $\langle s\rangle=0.167$. The time to fixation can be either faster (green) or slower (red) than the spatially homogeneous landscape with $\delta s=0$. Inset: selection landscapes for $\delta s=-0.2$ and $\delta s=0.5$.

slowed by heterogeneity - both timescales contribute to the dynamics. While we restrict ourselves primarily to $N=25,\langle s\rangle=1 / 6$, and to symmetric landscapes, we find qualitatively similar results (i.e. 3 distinct regions) for other values of $\langle s\rangle$ (Fig. S2), $N$ (Fig. S3), as well as for permuted selection profiles (Fig. S4), globally coupled profiles (Fig. S4), and monotonic (gradient) selection profiles (Fig. S5).

To intuitively understand these results, we developed a simple analytical approximation for $\tau_{f}$ (see SI, Equation S16) valid in the limit $\mu, \beta \ll 1$, where the fixation time is dominated by the arrival times of individual mutants (either from de novo mutation or from migration from a neighboring vial that has achieved fixation). In this limit, the three habitats achieve fixation one at a time, and fixation in a single habitat is approximated as an exponential process with rate $\lambda\left(s, n_{f i x}\right)=N\left(\mu+\beta n_{f i x}\right) P_{f i x}(s)$, where $n_{f i x}$ is the number of neighboring vials that have already achieved fixation and $P_{f i x}=s\left(1-(1-s)^{N}\right)^{-1}$ is the probability of a single mutant fixing in a habitat with selection pressure $s$ (see SI). The approximation captures the quali- tative features of fixation over a wide range of $\mu$ and $\beta$ (Fig. S7) and, in many cases, provides excellent quantitative agreement as well (see, for example, Fig. 2b, left panel and Fig. S7).

In general, the analytical approximation for $\tau_{f}$ is algebraically cumbersome. However, in the limit $\beta \ll \mu$, the approximation reduces to the expected maximum of three independent exponential random variables, leading to $\tau_{f} \approx \tau_{\max }=\lambda_{0}^{-1}+\lambda_{1}^{-1}+\lambda_{2}^{-1}-\left(\lambda_{0}+\lambda_{1}\right)^{-1}-\left(\lambda_{0}+\lambda_{2}\right)^{-1}-$ $\left(\lambda_{1}+\lambda_{2}\right)^{-1}+\left(\lambda_{0}+\lambda_{1}+\lambda_{2}\right)^{-1}$, with $\lambda_{i} \equiv \lambda\left(s\left(x_{i}\right), 0\right)$ (see SI for details). In this limit, the three-vial system acts effectively as three independent systems, with the overall fixation time corresponding to the slowest fixation. After rewriting $\tau_{\max }$ in terms of $\langle s\rangle$ and $\delta s$, it is straightforward to show that $\left.\left(\partial \tau_{\max } / \partial \delta s\right)\right|_{\delta s=0}=0$ and $\left.\left(\partial^{2} \tau_{\max } / \partial \delta s^{2}\right)\right|_{\delta s=0}>0$, indicating that the homogeneous landscape $(\delta s=0)$ minimizes the fixation time, consistent with results of the exact calculation (Fig. 2b, left panel). Intuitively, increasing heterogeneity reduces the minimum selection pressure in the spatial array, which in turn slows the expected maximum fixation time among the three habitats.

By contrast, in the limit $\mu \ll \beta, \tau_{f}$ reduces to the expected minimum of three independent exponential processes, leading to $\tau_{f} \approx \tau_{\text {min }}=\left(\lambda_{e f f}\right)^{-1}$, where $\lambda_{\text {eff }} \equiv$ $\lambda_{0}+\lambda_{1}+\lambda_{2}$. In this limit, the fixation time is dominated by dynamics in the vial that first achieves fixation; the remaining vials then rapidly achieve fixation due to fast migration. For large but finite $N$, the fixation time $\tau_{\min }$ is maximized at $\delta s=0$, indicating that heterogeneity always accelerates fixation, again consistent with the exact calculation (Fig. 2b, right panel). In this limit, the effective rate of fixation $\lambda_{\text {eff }}$ is increased for all $\delta \neq 0$, as heterogeneity decreases fixation time in the vial with the fastest average fixation.

Our results indicate that a judicious choice of selection pressure profile can potentially slow fixation of de novo mutants. In addition, selection pressure profiles can be optimized to mitigate the effects of resistance once it has emerged. One advantage of the MFPT approach (i.e. solving Equation 2) is that it provides fixation times starting from all possible initial states, making it straightforward to apply to cases where a resistant subpopulation already exists. Specifically, consider a situation where a resistant subpopulation has arisen at a particular spatial location. Is it possible to choose the spatial distribution of selection pressure - for example, by spatially dosing the drug - to minimize the time to fixation from this state? Intuitively, the goal is to delay the onset of treatment failure as long as possible. As an illustrative example, we consider a population consisting of $N / 2$ mutants in the center microhabitat and calculate the mean time to fixation for different spatial profiles of selection pressure. We then find the optimal value for $\delta s$ - that is, the heterogeneity corresponding to the spatial landscape with the slowest fixation time - in different 


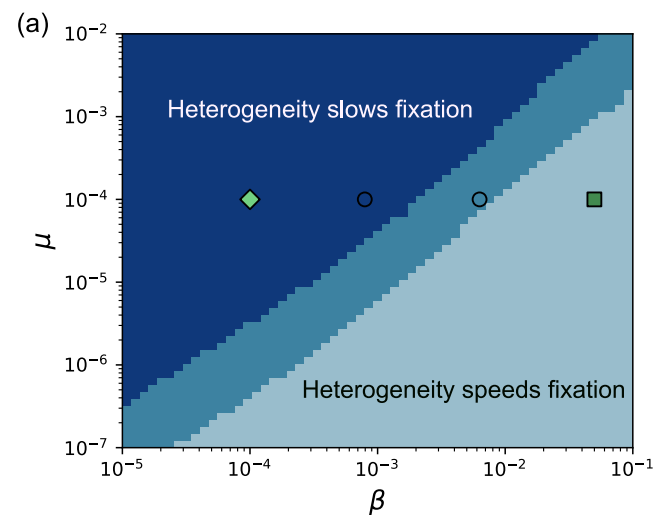

(b)

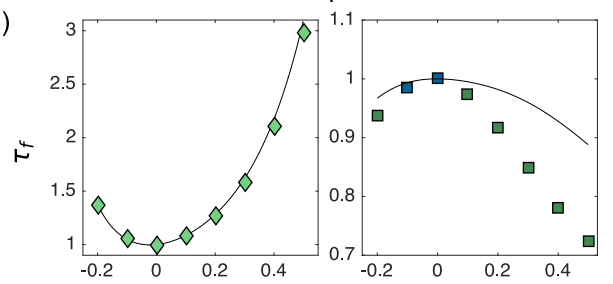

(c)

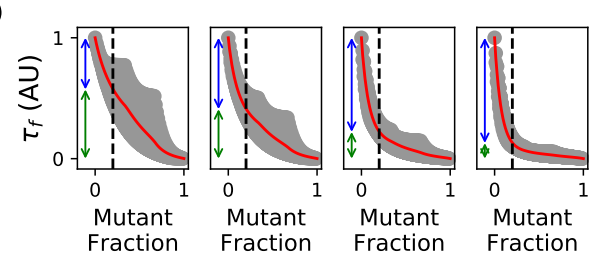

FIG. 2. Spatial heterogeneity can speed or slow fixation depending on the rates of migration $(\beta)$ and mutation $(\mu)$. (a) Phase diagram illustrates region of parameter space where the homogeneous landscape leads to a maximum (light blue), minimum (dark blue) or intermediate (medium blue) value of the in fixation time. MFPT calculations were performed for the indicated values of $\beta$ and $\mu$ and for $-0.2 \leq \delta s \leq 0.5$ in steps of 0.1. (b) Sample fixation curves in the regions where heterogeneity slows fixation (left panel, diamonds; $\beta=10^{-4}, \mu=10^{-4}$ ) or accelerates fixation (right panel, squares; $\left.\beta=5 \times 10^{-2}, \mu=10^{-4}\right)$. Solid curves indicate analytical approximations. (c) Gray shaded region indicates fixation time $\tau_{f}$ from every initial state $\left(n^{*}\left(x_{0}\right), n^{*}\left(x_{1}\right), n^{*}\left(x_{2}\right)\right)$, where $n^{*}\left(x_{i}\right)$ is the initial number of mutants at position $x_{i}$. Red curves show mean fixation time over all initial states with a given total mutant fraction. Vertical arrows represent time to achieve a total mutant fraction of $1 / 5\left(\tau_{1}\right.$, blue $)$ and time to go from that fraction to fixation $\left(\tau_{2}\right.$, green). Left to right panels: increasing $\beta$ at a fixed value of $\mu=10^{-4}$; plots correspond to symbols on phase diagram in panel (a). $N=25$ and $\langle s\rangle=0.167$ in all panels.

regions of parameter space (Fig. 3a). The specific choice of spatial profile significantly impacts the time to fixation from the initial resistant subpopulation (Fig. 3b). We observe two distinct regions of parameter space that lead to two very different dosing regimes (Fig. 3c). For $\mu$ sufficiently large relative to $\beta$, slowest fixation occurs when we maximize the amount of drug in the center microhabitat ( $\delta s=0.5$, white region). On the other hand, (a)

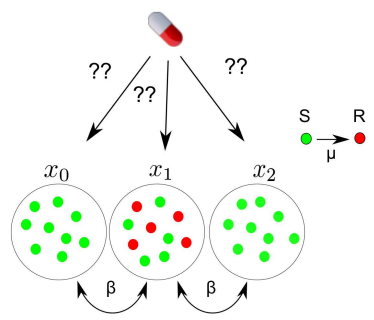

(c)

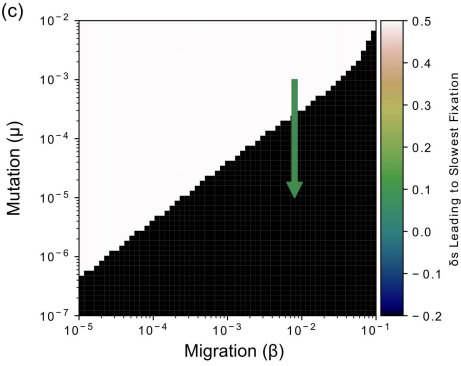

(b)

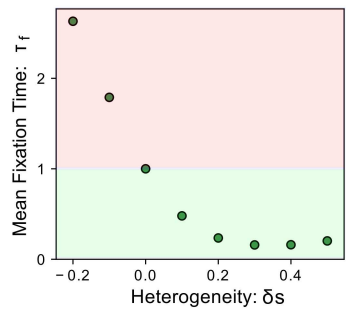

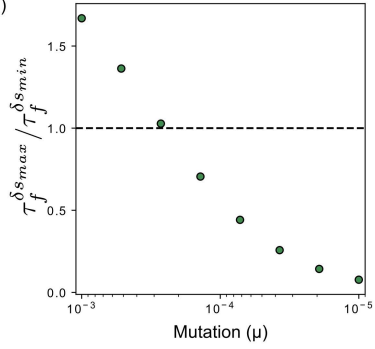

FIG. 3. (a) Schematic: a subpopulation of resistant mutants (red) arises at a particular spatial location. How can one choose the spatial distribution of selection pressure (i.e. drug concentration) to maximize the time to fixation? (b) Heterogeneity can significantly speed or slow fixation starting from an initial resistant subpopulation consisting of $N / 2$ cells in the center habitat $\left(\mu=10^{-5}, \beta=8 \times 10^{-3}\right)$. (c) The optimal spatial heterogeneity $(\delta s)$ leading to the slowest mean fixation time from an initial state of $(0, N / 2,0)$. Depending on the specific parameter regime, the optimal selection pressure profile is the one with the largest possible valley consistent with $\langle s\rangle$ (black) or the one having the largest possible peak (white). (d) Relative magnitude of $\tau_{f}^{\delta s_{\max }}$ (mean fixation time at maximum value of $\delta s$ ) and $\tau_{f}^{\delta s_{\min }}$ (mean fixation time at minimum value of $\delta s$ ) as mutation rate decreases at constant migration rate (green arrow, panel (c)). $N=25$ and $\langle s\rangle=0.167$ in all panels.

at large migration rates fixation is optimally slowed by maximizing the amount of drug in the two microhabitats without any initial mutants $(\delta s=-0.2)$. In contrast to the case with no initial mutants (e.g. Figure 2), fixation time is never maximized by choosing the homogeneous profile. To further characterize these two regimes, we compare the fixation times from a maximally peaked landscape ( $\delta s$ is maximized) to that from a landscape with a large valley ( $\delta s$ is minimized). The selection landscape that leads to the slowest fixation rapidly becomes sub-optimal as mutation rate is decreased at constant $\beta$ (Fig. 3d).

Our model is a dramatic oversimplification of the biological dynamics leading to drug resistance. Practical applications will require analysis of more realistic models and may call for spatial optimizations with different constraints-for example, limits on the maximum allowable local selection pressure. Nevertheless, the simplicity of our model allows for a thorough characterization of fixation time over a wide range of parameters, and its behavior is surprising rich. Importantly, our results do 
not require a fitness cost of resistance or a genetic fitness valley, and they predict that spatial heterogeneity in drug concentrations would impact populations of motile and non-motile cells in opposing ways, even when mutations rates are relatively similar. While heterogeneity is likely to accelerate evolution for populations of motile bacteria, similar to what is observed in experiments with E. coli 22, 24], our results predict slowed evolution for less motile cells (e.g. the nosocomial pathogen E. faecalis [49]) or cells with rapid mutation rates. Perhaps most interestingly, our results suggest counter-intuitive, spatially optimal profiles for slowing the spread of resistance sub-populations. In the long term, these results may lay the groundwork for optimized, spatially-resolved drug dosing strategies for mitigating the effects of drug resistance.

* kbwood@umich.edu

[1] S. B. Levy and B. Marshall, "Antibacterial resistance worldwide: causes, challenges and responses," Nature medicine, vol. 10, no. 12s, p. S122, 2004.

[2] C. Holohan, S. Van Schaeybroeck, D. B. Longley, and P. G. Johnston, "Cancer drug resistance: an evolving paradigm," Nature reviews. Cancer, vol. 13, no. 10, p. 714, 2013.

[3] M. M. Gottesman, "Mechanisms of cancer drug resistance," Annual review of medicine, vol. 53, no. 1, pp. 615-627, 2002.

[4] J. Davies and D. Davies, "Origins and evolution of antibiotic resistance," Microbiology and Molecular Biology Reviews, vol. 74, no. 3, pp. 417-433, 2010.

[5] M. Baym, L. K. Stone, and R. Kishony, "Multidrug evolutionary strategies to reverse antibiotic resistance," Science, vol. 351, no. 6268, p. aad3292, 2016.

[6] I. Bozic, J. G. Reiter, B. Allen, T. Antal, K. Chatterjee, P. Shah, Y. S. Moon, A. Yaqubie, N. Kelly, D. T. Le, et al., "Evolutionary dynamics of cancer in response to targeted combination therapy," Elife, vol. 2, p. e00747, 2013.

[7] Y. Iwasa, M. A. Nowak, and F. Michor, "Evolution of resistance during clonal expansion," Genetics, vol. 172, no. 4, pp. 2557-2566, 2006.

[8] E. Toprak, A. Veres, J.-B. Michel, R. Chait, D. L. Hartl, and R. Kishony, "Evolutionary paths to antibiotic resistance under dynamically sustained drug selection," $\mathrm{Na}$ ture genetics, vol. 44, no. 1, pp. 101-105, 2012.

[9] E. A. Yurtsev, H. X. Chao, M. S. Datta, T. Artemova, and J. Gore, "Bacterial cheating drives the population dynamics of cooperative antibiotic resistance plasmids," Molecular systems biology, vol. 9, no. 1, p. 683, 2013.

[10] R. Chait, A. Craney, and R. Kishony, "Antibiotic interactions that select against resistance," Nature, vol. 446, no. 7136, p. 668, 2007.

[11] J. P. Torella, R. Chait, and R. Kishony, "Optimal drug synergy in antimicrobial treatments," PLoS computational biology, vol. 6, no. 6, p. e1000796, 2010.

[12] E. Hansen, R. J. Woods, and A. F. Read, "How to use a chemotherapeutic agent when resistance to it threatens the patient," PLoS biology, vol. 15, no. 2, p. e2001110, 2017.

[13] A. Fischer, I. Vázquez-García, and V. Mustonen, "The value of monitoring to control evolving populations," Proceedings of the National Academy of Sciences, vol. 112, no. 4, pp. 1007-1012, 2015.

[14] H. Chung, T. D. Lieberman, S. O. Vargas, K. B. Flett, A. J. McAdam, G. P. Priebe, and R. Kishony, "Global and local selection acting on the pathogen stenotrophomonas maltophilia in the human lung," $\mathrm{Na}$ ture communications, vol. 8, p. 14078, 2017.

[15] D. Nichol, P. Jeavons, A. G. Fletcher, R. A. Bonomo, P. K. Maini, J. L. Paul, R. A. Gatenby, A. R. Anderson, and J. G. Scott, "Steering evolution with sequential therapy to prevent the emergence of bacterial antibiotic resistance," PLoS computational biology, vol. 11, no. 9, p. e1004493, 2015.

[16] M. Gralka, D. Fusco, S. Martis, and O. Hallatschek, "Convection shapes the trade-off between antibiotic efficacy and the selection for resistance in spatial gradients," Physical Biology, vol. 14, no. 4, p. 045011, 2017.

[17] F. Fu, M. A. Nowak, and S. Bonhoeffer, "Spatial heterogeneity in drug concentrations can facilitate the emergence of resistance to cancer therapy," PLoS Comput Biol, vol. 11, no. 3, p. e1004142, 2015.

[18] P. Greulich, B. Waclaw, and R. J. Allen, "Mutational pathway determines whether drug gradients accelerate evolution of drug-resistant cells," Physical Review Letters, vol. 109, no. 8, p. 088101, 2012.

[19] R. Hermsen, J. B. Deris, and T. Hwa, "On the rapidity of antibiotic resistance evolution facilitated by a concentration gradient," Proceedings of the National Academy of Sciences, vol. 109, no. 27, pp. 10775-10780, 2012.

[20] T. B. Kepler and A. S. Perelson, "Drug concentration heterogeneity facilitates the evolution of drug resistance," Proceedings of the National Academy of Sciences, vol. 95, no. 20, pp. 11514-11519, 1998.

[21] S. Moreno-Gamez, A. L. Hill, D. I. Rosenbloom, D. A. Petrov, M. A. Nowak, and P. S. Pennings, "Imperfect drug penetration leads to spatial monotherapy and rapid evolution of multidrug resistance," Proceedings of the $\mathrm{Na}$ tional Academy of Sciences, vol. 112, no. 22, pp. E2874E2883, 2015.

[22] Q. Zhang, G. Lambert, D. Liao, H. Kim, K. Robin, C.-k. Tung, N. Pourmand, and R. H. Austin, "Acceleration of emergence of bacterial antibiotic resistance in connected microenvironments," Science, vol. 333, no. 6050, pp. 1764-1767, 2011.

[23] R. Hermsen and T. Hwa, "Sources and sinks: a stochastic model of evolution in heterogeneous environments," Physical review letters, vol. 105, no. 24, p. 248104, 2010.

[24] M. Baym, T. D. Lieberman, E. D. Kelsic, R. Chait, R. Gross, I. Yelin, and R. Kishony, "Spatiotemporal microbial evolution on antibiotic landscapes," Science, vol. 353, no. 6304, pp. 1147-1151, 2016.

[25] C. S. Gokhale, Y. Iwasa, M. A. Nowak, and A. Traulsen, "The pace of evolution across fitness valleys," Journal of Theoretical Biology, vol. 259, no. 3, pp. 613-620, 2009.

[26] Y. Iwasa, F. Michor, and M. A. Nowak, "Stochastic tunnels in evolutionary dynamics," Genetics, vol. 166, no. 3, pp. 1571-1579, 2004.

[27] D. M. Weinreich, L. Chao, and P. Phillips, "Rapid evolutionary escape by large populations from local fitness peaks is likely in nature," Evolution, vol. 59, no. 6, 
pp. $1175-1182,2005$.

[28] D. B. Weissman, M. M. Desai, D. S. Fisher, and M. W. Feldman, "The rate at which asexual populations cross fitness valleys," Theoretical population biology, vol. 75, no. 4, pp. 286-300, 2009.

[29] S. T. Pickett and M. L. Cadenasso, "Landscape ecology: spatial heterogeneity in ecological systems," Science, vol. 269, no. 5222, p. 331, 1995.

[30] M. C. Whitlock and R. Gomulkiewicz, "Probability of fixation in a heterogeneous environment," Genetics, vol. 171, no. 3, pp. 1407-1417, 2005.

[31] L. A. Real and R. Biek, "Spatial dynamics and genetics of infectious diseases on heterogeneous landscapes," Journal of the Royal Society Interface, vol. 4, no. 16, pp. 935-948, 2007.

[32] V. Manem, M. Kohandel, N. Komarova, and S. Sivaloganathan, "Spatial invasion dynamics on random and unstructured meshes: Implications for heterogeneous tumor populations," Journal of Theoretical Biology, vol. 349, pp. $66-73,2014$.

[33] V. S. K. Manem, K. Kaveh, M. Kohandel, and S. Sivaloganathan, "Modeling invasion dynamics with spatial random-fitness due to micro-environment," PLOS ONE, vol. 10, pp. 1-20, 102015.

[34] S. Farhang-Sardroodi, A. Darooneh, M. Nikbakht, N. Komarova, and M. Kohandel, "The effect of spatial randomness on the average fixation time of mutants.," PLoS computational biology, vol. 13, no. 11, p. e1005864, 2017.

[35] A. Kaznatcheev, J. G. Scott, and D. Basanta, "Edge effects in game-theoretic dynamics of spatially structured tumours," Journal of The Royal Society Interface, vol. 12, no. 108, p. 20150154, 2015.

[36] B. Allen, G. Lippner, Y.-T. Chen, B. Fotouhi, N. Momeni, S.-T. Yau, and M. A. Nowak, "Evolutionary dynamics on any population structure," Nature, vol. 544, no. 7649, pp. 227-230, 2017.

[37] K. S. Korolev, M. Avlund, O. Hallatschek, and D. R. Nelson, "Genetic demixing and evolution in linear stepping stone models," Reviews of modern physics, vol. 82, no. 2, p. 1691, 2010.

[38] M. Khasin, E. Khain, and L. M. Sander, "Fast migration and emergent population dynamics," Physical review letters, vol. 109, no. 24, p. 248102, 2012.

[39] M. Khasin, B. Meerson, E. Khain, and L. M. Sander, "Minimizing the population extinction risk by migration," Physical review letters, vol. 109, no. 13, p. 138104, 2012.

[40] J. N. Waddell, L. M. Sander, and C. R. Doering, "Demographic stochasticity versus spatial variation in the competition between fast and slow dispersers," Theoretical population biology, vol. 77, no. 4, pp. 279-286, 2010.

[41] Y. T. Lin, H. Kim, and C. R. Doering, "Demographic stochasticity and evolution of dispersion i. spatially homogeneous environments," Journal of Mathematical Biology, vol. 70, no. 3, pp. 647-678, 2015.

[42] Y. T. Lin, H. Kim, and C. R. Doering, "Demographic stochasticity and evolution of dispersion ii: Spatially inhomogeneous environments," Journal of Mathematical Biology, vol. 70, pp. 679-707, Feb 2015.

[43] G. W. Constable and A. J. McKane, "Fast-mode elimination in stochastic metapopulation models," Physical Review E, vol. 89, no. 3, p. 032141, 2014.

[44] G. W. Constable and A. J. McKane, "Population genetics on islands connected by an arbitrary network: An analytic approach," Journal of theoretical biology, vol. 358, pp. 149-165, 2014.

[45] G. W. Constable and A. J. McKane, "Stationary solutions for metapopulation moran models with mutation and selection," Physical Review E, vol. 91, no. 3, p. $032711,2015$.

[46] C. Li, "The statistical processes of evolutionary theory," American journal of human genetics, vol. 14, no. 4, p. $438,1962$.

[47] C. W. Gardiner, Handbook of stochastic methods for physics, chemistry and the natural sciences, vol. 13 of Springer Series in Synergetics. Berlin: Springer-Verlag, third ed., 2004.

[48] N. V. Kampen, Stochastic processes in physics and chemistry. North Holland, 2007.

[49] D. B. Clewell, M. S. Gilmore, Y. Ike, and N. Shankar, Enterococci: from commensals to leading causes of drug resistant infection. Massachusetts Eye and Ear Infirmary, 2014. 